$\xi^{2}=-1$

\title{
Assessment of groundwater quality by using statistical analysis from kopargaon taluka, Ahmednagar, India
}

\author{
Deshpande A.V. *, Patil S.N. \\ Department of Applied Geology, School of Environmental and Earth Sciences, North Maharashtra University, Jalgaon(India) 425001 \\ *Corresponding author E-mail: manjushamnil@gmail.com
}

\begin{abstract}
The study was conducted to evaluate the groundwater quality along the Kopargaon taluka. Thirty six ground water samples were collected from different sources in pre monsoon and post monsoon season, during the year 2013. The descriptive statistical analysis was carried

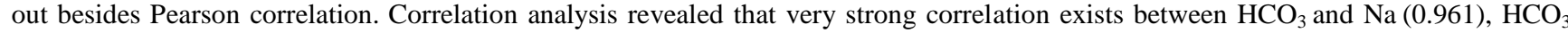
and $\mathrm{Mg}^{++}(0.935), \mathrm{HCO}_{3}$ and $\mathrm{EC}(0.927)$. Where highly negative correlation is observed between $\mathrm{Na}$ and $\mathrm{pH}(-0.537)$ during premonsoon season. During post monsoon season highly positive correlation is observed between $\mathrm{Cl}^{-}$and $\mathrm{Ca}^{++}(0.973)$, $\mathrm{Ca}$ and $\mathrm{EC}(0.967)$, $\mathrm{Cl}^{-}$and $\mathrm{EC}(0.966), \mathrm{SO}_{4}$ and $\mathrm{EC}(0.948)$. Where, highly negative correlation is observed between $\mathrm{Ca}$ and $\mathrm{pH}(-0.533)$.
\end{abstract}

Keywords: Groundwater; Physico-Chemical Parameter; Statistical Analysis; Correlation Analysis.

\section{Introduction}

Groundwater is considered as the major source of usable water, so that quality of water is the main key factor in management of groundwater in a sustainable manner. In the past few decades, reports of groundwater contamination have increased public concern; reports of ground water contamination have increased public concern about ground water quality (Kant et al 2015). In agro based industries, sugar cane industry plays important role in India. This sugar cane industry plays vital role in foreign exchange and helps Indian economy. The wastewater of these industries pollutes the water which becomes the threat for the environment and also affects the soil. In spite of the fact that sugar industry is the backbone of the rural economy of Maharashtra state the need has arisen to review and recognize environmental problem associated with it. , the sugar factory is a rural industry and continued operation of this industry, without environmental precautions, may lead to serious health problems in the area. In the Ahmednagar district alone, there are 13 such sugar factories with several types of allied units associated with them. Moreover, in majority of villages there is no organized water-supply facility. Instead, the rural population is dependent on traditional sources of water supply. Disposal of industrial waste is the major cause of soil \& water pollution. Correlation coefficient is used to measure the strength of association between two continuous variables. This tells if the relation between the variables is positive or negative that is one increase with the increase of the other. Thus, the correlation measures the observed co-variation. The most commonly used measure of correlation is Pearson's correlation (r). It is also called the linear correlation coefficient because $r$ measures the linear association between two variables (Khwaja and Agrawal, 2014).Description of the study area

The study area is situated in Ahmednagar district. Ahmednagar district lies between $18^{\circ} 02^{\prime}$ and $19^{\circ} 09^{\prime}$ North latitudes and $73^{\circ} 09^{\prime}$ and $75^{\circ} 05^{\prime}$ 'East longitudes. The district is bounded on the north by the districts of Nasik and Aurangabad, on the east by Beed \&
Osmanabad, on the south by Solapur and on the west by Thane and Pune Districts. Ahmednagar district covers an area of about 17,0448 sq.km. Area wise largest district in the state covering $5.55 \%$ area of the total area of Maharashtra state. The location map of study area is shown in figure 1 .

\subsection{Hydrogeology}

The major part of the district is underlain by the basaltic lava flows, which were formed by the intermittent fissure type eruptions during of upper Cretaceous to lower Eocene age. The Deccan Trap has succession of 19 major flows in the elevation range of 420 to $730 \mathrm{~m}$ above mean sea level. These flows are characterized by the prominent units of vesicular and massive Basalt. The Alluvium of Recent age also occurs as narrow stretch along the course of major rivers deposited over the Traps. A map depicting the hydrogeological features is shown in figure 3 .

\subsection{Geomorphology and soil types}

Physiographically the district forms part of Deccan Plateau. Part of Sahayadri hill ranges fall in the district. Western Ghat section in Akole taluka is hilly which extends to relatively flat areas in Shevgaon and Jamkhed talukas in the east. From the main Sahayadri range three spurs namely Kalsubai, Baleshwar and Hari shchandragad stretch eastwards. Physiographically the district can be broadly divided in four major characteristic landforms viz., hill and ghat section ( $7.6 \%$ area); foothill zone (19.4\% area); plateau (3.71\% area) and plains (occupy $69.30 \%$ area). The district lies partly in Godavari basin and partly in Bhima basin. The northern part of the district is drained by Godavari River and its tributaries viz., Pravara, Mula, Adula and Mahalungi whereas the southern part is drained by Bhima River and its tributaries viz., Ghod and Sina. All the rivers have sub-parallel to semi-dendritic drainage pattern and the drainage density is quite high. Based on geomorphological setting and drainage pattern, the district is divided into 80 watershed 


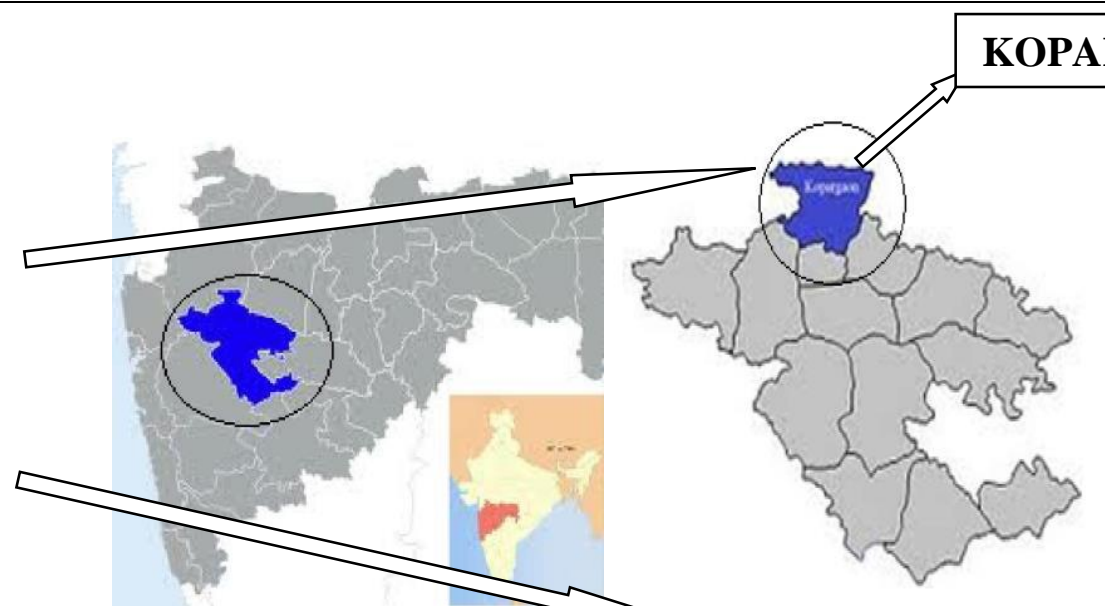

Fig. 1: Location norp cutudy Area.

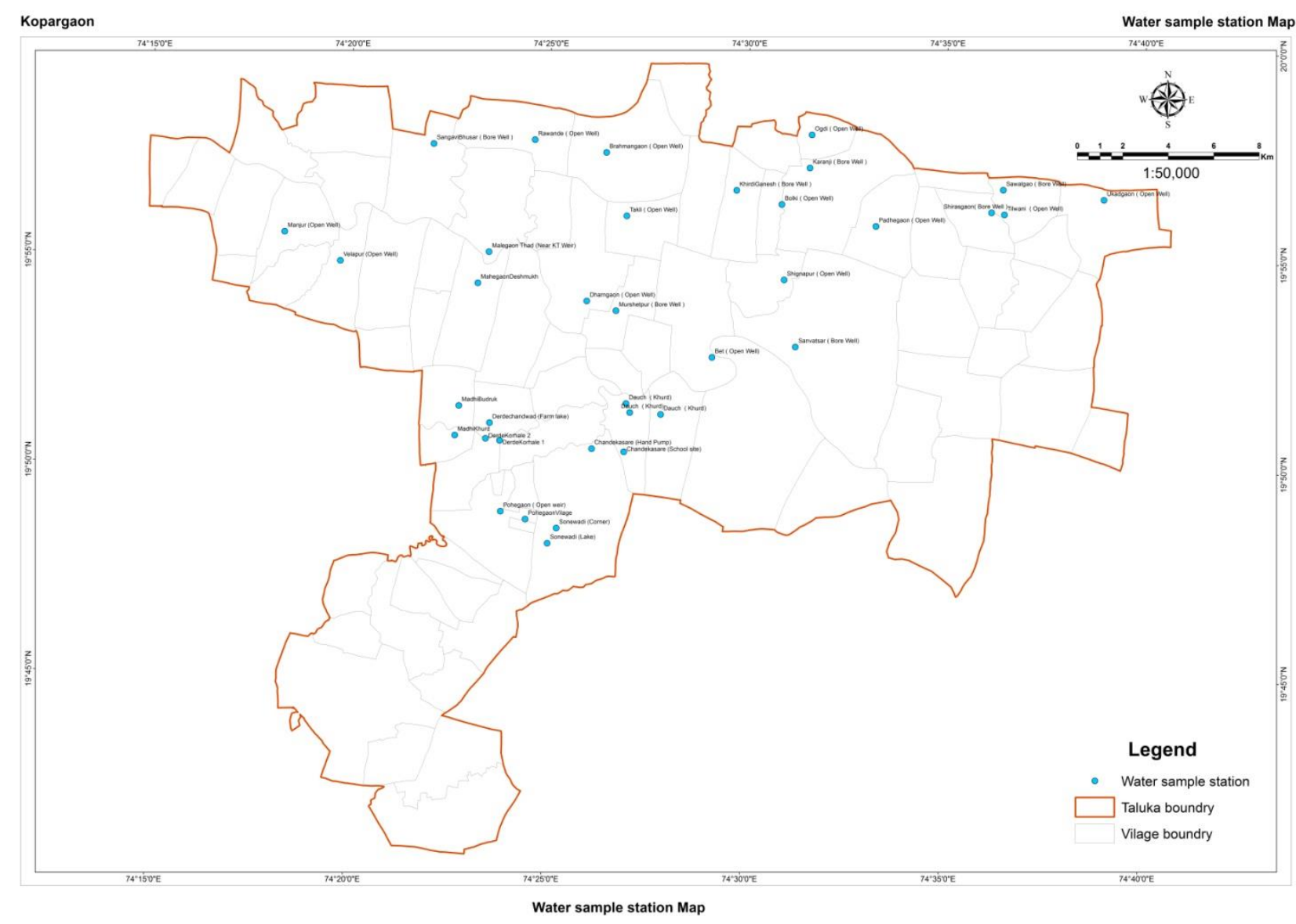

Fig. 2: Over View of Sampling Locations. 


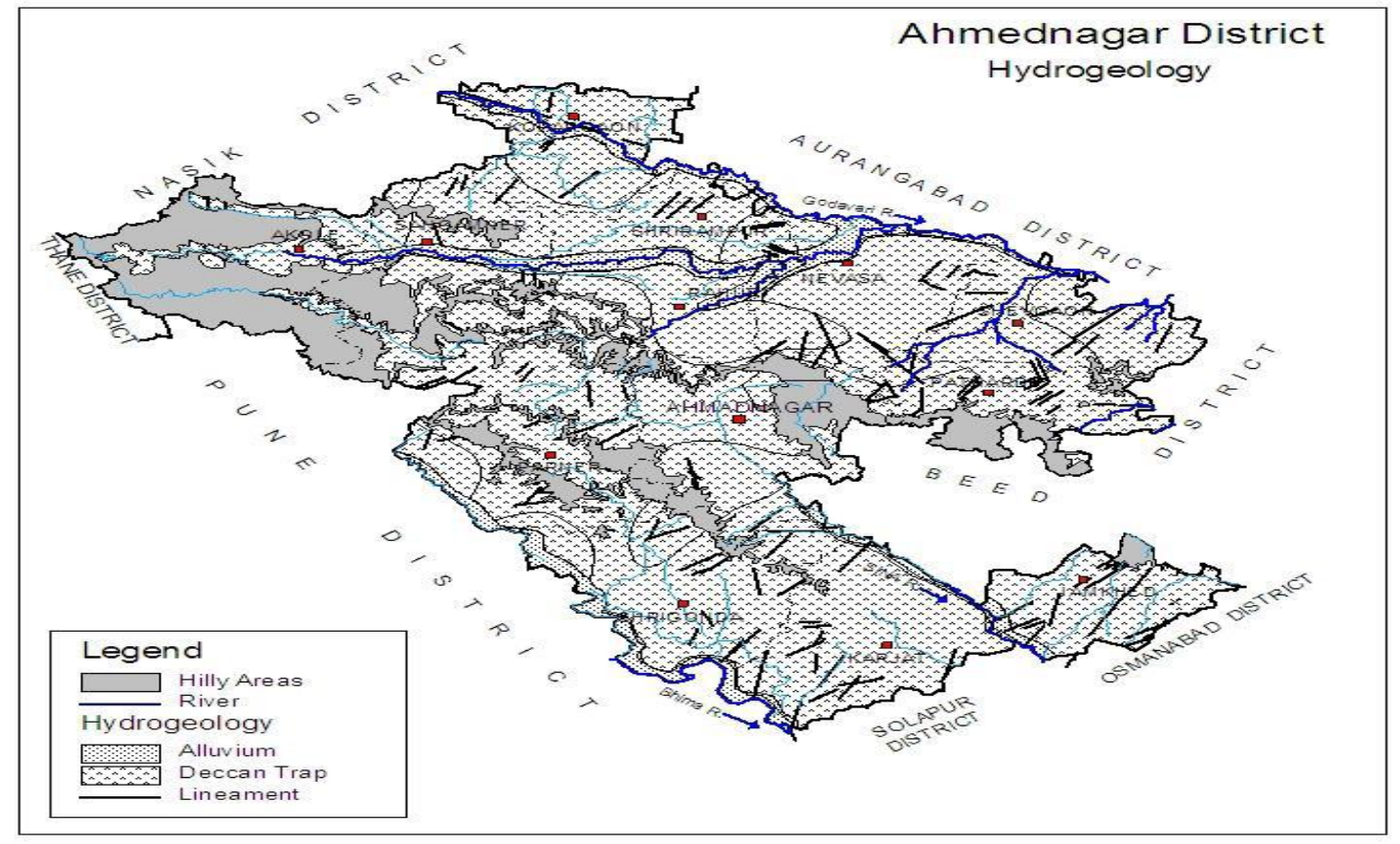

Fig. 3: Hydrogeological Map of the Study Area (CGWB 2014).

\section{Materials and methodology}

Ground water was sampled from 36 different stations during the pre-monsoon and post monsoon seasons in the year 2013. These collected samples were analyzed for water quality parameters viz. $\mathrm{pH}$, Electrical conductivity (EC), Calcium, Magnesium, Sodium, Potassium, Ferrous, Manganese, Copper, Zink, Nickel, Chloride, Sulphate etc. All the tests were conducted in accordance with the techniques described by American Public Health (APHA 2005).Various statistical analysis of the experimental data were performed using Microsoft Excel 2010. The results of physicochemical parameters as determined in samples collected from Kopargaon Taluka. The results of analysis obtained are summarized in Table 3. Normal statistic of water quality parameters of groundwater samples (pre-monsoon season) are summarized in Table 4.

Table 2: Instrumental Methods for Measurement of Different Water Quality Parameters

\begin{tabular}{ll}
\hline Parameter & Equipment \\
\hline $\mathrm{pH}$ & Digital pH meter \\
Electrical conductivity & Electrical conductivity meter \\
Total hardness & Titrimetric method (EDTA) \\
Calcium & Flame- photometric method \\
Magnesium & Flame- photometric method \\
Chloride & color indicator titrimetric method \\
Sodium & Flame- photometric method \\
Potassium & Flame- photometric method \\
Sulphate & Digital Spectrophotometer \\
\hline
\end{tabular}

\section{Result and discussion}

\subsection{Physico-chemical analysis of ground water quality parameters during pre-monsoon season}

Ground water samples were collected from Kopargaon area. These samples were collected during summer season .The results of analysis are tabulated below in Table 3

In Pre- monsoon: Table 5shows highly positive correlation is observed between $\mathrm{HCO}_{3}$ and $\mathrm{Na}(0.961), \mathrm{HCO}_{3}$ and $\mathrm{Mg}^{++}(0.935)$, $\mathrm{HCO}_{3}$ and $\mathrm{EC}(0.927)$. Where highly negative correlation is observed between $\mathrm{Na}$ and $\mathrm{pH}(-0.538)$. Very poor positive correlation was observed between $\mathrm{Fe}$ and $\mathrm{pH}(-0.0008)$. While, there is almost no correlation was observed between $\mathrm{Ni}$ and $\mathrm{Ca}(0.004)$.

\subsection{Physico-chemical analysis of ground water quality parameters during post-monsoon season}

Ground water samples were collected from Kopargaon area. These samples were collected during post-monsoon season .The results of analysis are tabulated below.

Table 3: Physico-Chemical Analysis of Ground Water Quality Parameters during Pre-Monsoon Season

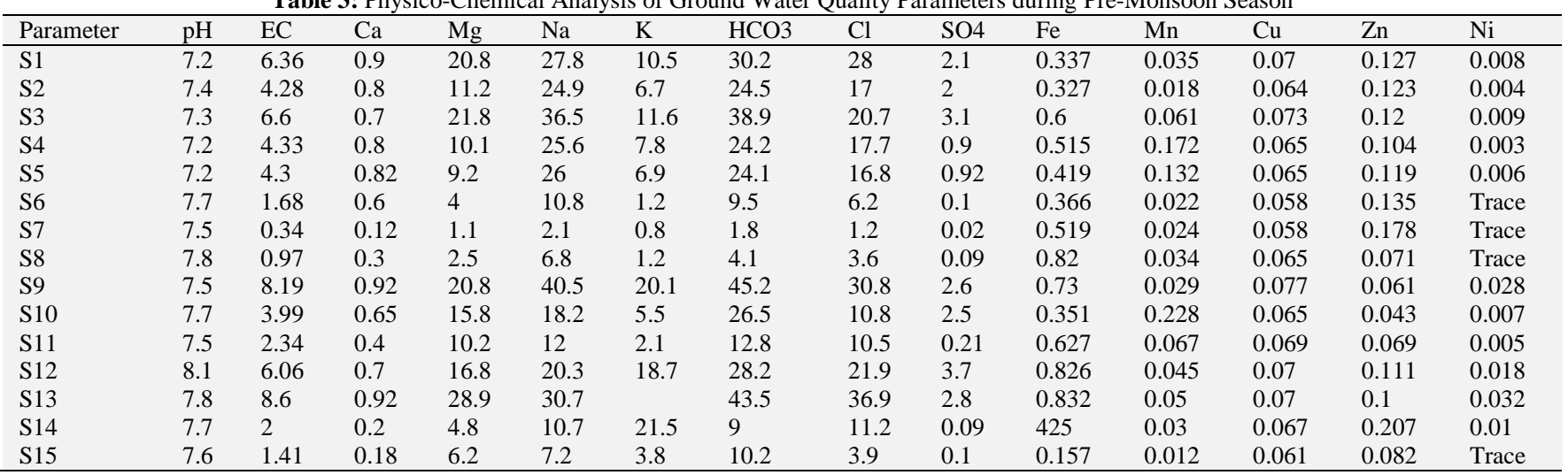




\begin{tabular}{|c|c|c|c|c|c|c|c|c|c|c|c|c|c|c|}
\hline S16 & 7.5 & 5.5 & 0.7 & 18.5 & 31.2 & 1.1 & 27.8 & 26 & 0.4 & 0.173 & 0.013 & 0.077 & 0.074 & 0.014 \\
\hline S17 & 7.6 & 1.5 & 0.14 & 6 & 8.2 & 6.9 & 8.5 & 7 & 0.08 & 0.114 & 0.009 & 0.054 & 0.073 & Trace \\
\hline S18 & 7.4 & 5.04 & 0.7 & 24.8 & 22.5 & 1.8 & 30.1 & 18.9 & 0.1 & 0.212 & 0.008 & 0.069 & 0.059 & 0.011 \\
\hline S19 & 7.5 & 8.2 & 0.94 & 30.1 & 42.1 & 5.1 & 48.5 & 30.1 & 3 & 0.18 & 0.035 & 0.081 & 0.086 & 0.032 \\
\hline S20 & 7.4 & 1.94 & 0.18 & 4.74 & 11.9 & 7.8 & 11.2 & 7.9 & 0.08 & 0.127 & 0.006 & 0.061 & 0.068 & Trace \\
\hline S21 & 7.9 & 4.12 & 0.32 & 11.9 & 20.1 & 3.2 & 18.7 & 16 & 0.73 & 0.235 & 0.009 & 0.06 & 0.073 & 0.016 \\
\hline S22 & 8.2 & 1.3 & 0.4 & 5.7 & 8.6 & 8 & 6.5 & 5.2 & 1.1 & 0.111 & 0.004 & 0.064 & 0.19 & Trace \\
\hline S23 & 7.7 & 1.95 & 0.5 & 5.9 & 10.5 & 1.1 & 11.1 & 8 & 0.7 & 0.132 & 0.005 & 0.06 & 0.98 & Trace \\
\hline S24 & 7.8 & 1.95 & 0.52 & 6 & 10.4 & 3.1 & 11.3 & 7.8 & 0.72 & 0.162 & 0.006 & 0.069 & 0.078 & Trace \\
\hline S26 & 7.8 & 3.39 & 0.7 & 12.9 & 13.4 & 6.2 & 20.1 & 11.2 & 1.4 & 0.118 & 0.005 & 0.073 & 0.062 & 0.011 \\
\hline S27 & 7.7 & 3.39 & 0.8 & 18.7 & 20.5 & 8.9 & 24.5 & 20.9 & 0.9 & 0.123 & 0.002 & 0.07 & 0.113 & 0.02 \\
\hline S28 & 8.1 & 4.81 & 0.3 & 4.2 & 6.8 & 1.1 & 3 & 7.1 & 1 & 0.151 & 0.009 & 0.067 & 0.049 & Trace \\
\hline S29 & 7.9 & 1.23 & 0.4 & 7 & 12.1 & 2.5 & 10.1 & 12.5 & 0.08 & 0.111 & 0.001 & 0.066 & 0.033 & 0.08 \\
\hline S30 & 8 & 2.23 & 0.25 & 6.8 & 3.4 & 0.9 & 5.2 & 5 & 0.1 & 0.098 & 0.001 & 0.062 & 0.078 & Trace \\
\hline S31 & 8 & 1.41 & 0.24 & 6.7 & 5.7 & 1.2 & 5.9 & 6.2 & 1.2 & 0.058 & 0.001 & 0.067 & 0.118 & Trace \\
\hline S32 & 8 & 1.11 & 0.24 & 5 & 4.5 & 1 & 5 & 4.9 & 1.1 & 0.1 & 0.008 & 0.06 & 0.087 & Trace \\
\hline S33 & 8.6 & 1.78 & 0.5 & 4.9 & 5.8 & 1.4 & 5.2 & 11.1 & 1.4 & 0.105 & 0.005 & 0.061 & 0.109 & Trace \\
\hline S35 & 7.7 & 1.5 & 0.22 & 6.2 & 7.9 & 1.1 & 3.8 & 9 & 1.2 & 0.035 & 0.002 & 0.057 & 0.083 & Trace \\
\hline S36 & 7.7 & 4.07 & 0.72 & 14.9 & 20.5 & 6.2 & 18.5 & 15.1 & 2.7 & 0.069 & 0.007 & 0.066 & 0.066 & 0.24 \\
\hline
\end{tabular}

Note: All units are in $\mathrm{mg} / \mathrm{l}$ except $\mathrm{pH} \& \mathrm{EC}$

Table 4: Normal Statistic of Water Quality Parameters of Groundwater Samples (Pre-Monsoon Season)

\begin{tabular}{lllllllllllllll}
\hline Parameter & $\mathrm{pH}$ & $\mathrm{EC}$ & $\mathrm{Ca}$ & $\mathrm{Mg}$ & $\mathrm{Na}$ & $\mathrm{K}$ & $\mathrm{HCO} 3$ & $\mathrm{Cl}$ & $\mathrm{SO}$ & $\mathrm{Fe}$ & $\mathrm{Mn}$ & $\mathrm{Cu}$ & $\mathrm{Zn}$ & $\mathrm{Ni}$ \\
\hline MIN & 7.2 & 0.34 & 0.12 & 1.1 & 2.1 & 0.8 & 1.8 & 1.2 & 0.02 & 0.035 & 0.001 & 0.054 & 0.033 & 0.003 \\
MAX & 8.6 & 8.6 & 0.94 & 30.1 & 42.1 & 21.5 & 48.5 & 36.9 & 3.7 & 425 & 0.228 & 0.081 & 0.98 & 0.24 \\
AVG & 7.7 & 3.451 & 0.531 & 11.323 & 16.553 & 5.628 & 17.71 & 13.83 & 1.148 & 12.086 & 0.030 & 0.065 & 0.120 & 0.028 \\
MED & 7.7 & 2.945 & 0.56 & 8.5 & 12.05 & 4.1 & 12.3 & 11.2 & 0.91 & 0.176 & 0.009 & 0.065 & 0.086 & 0.012 \\
SD & 0.3125 & 2.229 & 0.260 & 7.599 & 10.671 & 5.431 & 12.78 & 8.696 & 1.044 & 70.78 & 0.049 & 0.006 & 0.152 & 0.041 \\
\hline
\end{tabular}

MIN- Minimum, MAX- Maximum, AVG-Average, MED Median, SD- Standard deviation

Table 5: Correlation Matrix of Water Quality Parameters (Pre-Monsoon Season)

\begin{tabular}{|c|c|c|c|c|c|c|c|c|c|c|c|c|c|c|}
\hline Parameter & $\mathrm{Ph}$ & $\mathrm{EC}$ & $\mathrm{Ca}$ & $\mathrm{Mg}$ & $\mathrm{Na}$ & K & $\mathrm{HC} 03$ & $\mathrm{CI}$ & $\mathrm{SO}_{4}$ & $\mathrm{Fe}$ & $\mathrm{Mn}$ & $\mathrm{Cu}$ & $\mathrm{Zn}$ & $\mathrm{Ni}$ \\
\hline $\mathrm{pH}$ & 1 & & & & & & & & & & & & & \\
\hline EC & -0.33981 & 1 & & & & & & & & & & & & \\
\hline $\mathrm{Ca}$ & -0.35033 & 0.79387 & 1 & & & & & & & & & & & \\
\hline $\mathrm{Mg}$ & -0.35701 & 0.891386 & 0.783346 & 1 & & & & & & & & & & \\
\hline $\mathrm{Na}$ & -0.53775 & 0.90607 & 0.832361 & 0.86452 & 1 & & & & & & & & & \\
\hline $\mathrm{K}$ & -0.2104 & 0.474794 & 0.336593 & 0.344919 & 0.46746 & 1 & & & & & & & & \\
\hline $\mathrm{HC} 03$ & -0.46959 & 0.927398 & 0.842634 & 0.934807 & 0.96084 & 0.493368 & 1 & & & & & & & \\
\hline $\mathrm{CI}$ & -0.33927 & 0.917963 & 0.828901 & 0.899029 & 0.906606 & 0.499071 & 0.914682 & 1 & & & & & & \\
\hline $\mathrm{Fe}$ & -0.0008 & -0.11008 & -0.21677 & -0.14632 & -0.09272 & 0.509664 & -0.11565 & -0.05063 & -0.17244 & 1 & & & & \\
\hline $\mathrm{Mn}$ & -0.36841 & 0.261768 & 0.327853 & 0.186956 & 0.3152 & 0.182708 & 0.334946 & 0.170351 & 0.264841 & -0.00102 & 1 & & & \\
\hline $\mathrm{Cu}$ & -0.21048 & 0.73075 & 0.652941 & 0.731119 & 0.728883 & 0.374844 & 0.739268 & 0.702957 & 0.506255 & 0.037007 & 0.111606 & 1 & & \\
\hline $\mathrm{Zn}$ & -0.00582 & -0.15083 & -0.3532 & -0.16241 & -0.11687 & -0.05444 & -0.11709 & -0.13094 & -0.07663 & 0.097481 & -0.0975 & -0.19528 & 1 & \\
\hline $\mathrm{Ni}$ & 0.205779 & -0.07434 & 0.00434 & -0.00287 & -0.06165 & -0.08974 & -0.13 & -0.0656 & 0.204307 & 0.08061 & -0.24907 & -0.06553 & -0.2767 & 1 \\
\hline
\end{tabular}

Table 6: Physico-Chemical Analysis of Ground Water Quality Parameters during Post-Monsoon Season

\begin{tabular}{|c|c|c|c|c|c|c|c|c|c|c|c|c|c|c|}
\hline Parameter & $\mathrm{pH}$ & EC & $\mathrm{Ca}$ & $\mathrm{Mg}$ & $\mathrm{Na}$ & $\mathrm{K}$ & $\mathrm{HCO}_{3}$ & $\mathrm{Cl}$ & $\mathrm{SO}_{4}$ & $\mathrm{Fe}$ & $\mathrm{Mn}$ & $\mathrm{Cu}$ & $\mathrm{Zn}$ & $\mathrm{Ni}$ \\
\hline S1 & 7.65 & 4.44 & 26.1 & 16.2 & 1.8 & 0.18 & 6.7 & 25.7 & 12 & 0.244 & 0.09 & 0.077 & 0.017 & 0.038 \\
\hline $\mathrm{S} 2$ & 7.82 & 1.19 & 6.2 & 3.8 & 0.89 & 0.1 & 1.1 & 5.2 & 4.9 & 0.236 & 0.016 & 0.032 & 0.0015 & 0.042 \\
\hline S3 & 7.46 & 6.13 & 35 & 22.1 & 1.76 & 0.81 & 7.2 & 34.5 & 19.8 & 0.255 & 0.01 & 0.058 & 0.021 & 0.063 \\
\hline S4 & 7.66 & 3.5 & 19.8 & 12.5 & 1.5 & 0.45 & 4.7 & 20.2 & 10.8 & 0.229 & 0.02 & 0.035 & 0.092 & 0.047 \\
\hline S5 & 8.1 & 1.64 & 10.2 & 4.9 & 0.82 & 0.2 & 2.6 & 7.8 & 4.5 & 0.233 & 0.017 & 0.016 & 0.047 & 0.049 \\
\hline S6 & 7.84 & 1.4 & 9.8 & 3.4 & 0.9 & 0.15 & 2.1 & 6.2 & 4.1 & 0.127 & 0.012 & 0.015 & 0.055 & 0.047 \\
\hline S7 & 8.16 & 0.42 & 3 & 1.2 & 0.25 & 0.09 & 1 & 2.1 & 1.9 & 0.129 & 0.028 & 0.014 & 0.038 & 0.02 \\
\hline S8 & 7.99 & 0.81 & 4.2 & 2.9 & 0.1 & 0.12 & 0.7 & 6.2 & 2 & 0.245 & 0.009 & 0.008 & 0.025 & 0.02 \\
\hline S10 & 7.11 & 2.66 & 15.1 & 8.1 & 1.1 & 0.7 & 4.4 & 12.7 & 10.1 & 0.24 & 0.005 & 0.016 & 0.057 & 0.044 \\
\hline S11 & 7.63 & 1.89 & 10.7 & 6.2 & 0.8 & 0.5 & 3.5 & 7.2 & 6.9 & 0.23 & 0.004 & 0.01 & 0.093 & 0.024 \\
\hline $\mathrm{S} 12$ & 8.3 & 0.66 & 3.1 & 2 & 0.4 & 0.15 & 1.2 & 2.4 & 1.1 & 0.145 & 0.013 & 0.013 & 0.052 & 0.061 \\
\hline S13 & 7.18 & 4.48 & 25.2 & 17 & 1.2 & 0.21 & 6.9 & 23.8 & 11.1 & 0.146 & 0.06 & 0.025 & 0.047 & 0.067 \\
\hline S14 & 7.14 & 2.49 & 11.9 & 10.1 & 0.7 & 0.24 & 3.4 & 12.2 & 7.2 & 0.15 & 0.014 & 0.013 & 0.052 & 0.061 \\
\hline S15 & 7.93 & 1.16 & 7.2 & 3.1 & 0.25 & 0.12 & 1.2 & 6.7 & 3.5 & 0.137 & 0.012 & 0.013 & 0.093 & 0.044 \\
\hline S16 & 7.13 & 1.17 & 8 & 2.9 & 0.2 & 0.15 & 1.4 & 6.6 & 3.4 & 0.232 & 0.012 & 0.007 & 0.027 & 0.048 \\
\hline S17 & 8.08 & 1.64 & 9 & 5.1 & 0.1 & 0.12 & 3.4 & 8.1 & 4 & 0.227 & 0.002 & 0.015 & 0.062 & 0.059 \\
\hline S19 & 7.45 & 1.01 & 6.1 & 2.9 & 0.08 & 0.1 & 2.8 & 4.2 & 3.1 & 0.267 & 0.017 & 0.017 & 0.084 & 0.117 \\
\hline S20 & 7.12 & 1.52 & 11 & 4.2 & 0.1 & 0.12 & 3 & 8.1 & 4.2 & 0.267 & 0.011 & 0.031 & 0.054 & 0.128 \\
\hline $\mathrm{S} 21$ & 7.82 & 3.34 & 22.1 & 6.4 & 2.1 & 0.6 & 5.2 & 20.1 & 8.1 & 0.246 & 0.02 & 0.01 & 0.029 & 0.064 \\
\hline S22 & 7.9 & 2.38 & 14.9 & 6.2 & 0.9 & 0.2 & 3.4 & 12.1 & 8.9 & 0.232 & 0.025 & 0.012 & 0.049 & 0.036 \\
\hline $\mathrm{S} 23$ & 7.64 & 2.58 & 20.1 & 4.2 & 0.5 & 0.25 & 4 & 14.2 & 7.1 & 0.25 & 0.017 & 0.05 & 0.097 & 0.077 \\
\hline S24 & 7.18 & 1.86 & 14.5 & 3.2 & 0.7 & 0.14 & 3.9 & 9.5 & 4.2 & 0.24 & 0.004 & 0.012 & 0.051 & 0.042 \\
\hline S25 & 8.16 & 1.44 & 7.9 & 4.1 & 2.58 & 0.2 & 3.2 & 6.2 & 4.1 & 0.235 & 0.02 & 0.02 & 0.027 & 0.053 \\
\hline S26 & 7.11 & 2.81 & 18.1 & 9.8 & 0.7 & 0.19 & 4.8 & 19.2 & 6.3 & 0.238 & 0.03 & 0.08 & 0.071 & 0.06 \\
\hline S27 & 7.88 & 2.81 & 20.5 & 8.2 & 0.98 & 0.21 & 5.7 & 20.1 & 4.9 & 0.246 & 0.016 & 0.01 & 0.016 & 0.089 \\
\hline S28 & 7.95 & 1.94 & 14.2 & 4.9 & 0.95 & 0.25 & 2.1 & 12.5 & 6.2 & 0.25 & 0.007 & 0.01 & 0.07 & 0.046 \\
\hline S29 & 7.88 & 2.25 & 15.2 & 5.7 & 1.28 & 0.5 & 3.1 & 16.1 & 4.2 & 0.248 & 0.015 & 0.24 & 0.058 & 0.083 \\
\hline
\end{tabular}




\begin{tabular}{lllllllllllllll}
\hline S30 & 7.81 & 1.55 & 7.5 & 8.1 & 0.8 & 0.1 & 2.9 & 8.1 & 4.1 & 0.134 & 0.009 & 0.09 & 0.1 & 0.034 \\
S31 & 7.83 & 1.17 & 7 & 3.5 & 0.7 & 0.2 & 3.2 & 6.1 & 2.9 & 0.133 & 0.006 & 0.018 & 0.066 & 0.072 \\
S32 & 7.89 & 2 & 14 & 5.1 & 0.5 & 0.21 & 4 & 12.1 & 7.2 & 0.138 & 0.014 & 0.011 & 0.081 & 0.068 \\
S33 & 8.13 & 1.79 & 6 & 5.1 & 2.95 & 0.28 & 8.9 & 5.7 & 4.2 & 0.187 & 0.013 & 0.004 & 0.111 & 0.052 \\
S34 & 7.56 & 3.03 & 20.1 & 9.2 & 0.95 & 0.7 & 6 & 16.1 & 8.1 & 0.144 & 0.014 & 0.05 & 0.066 & 0.096 \\
S35 & 8.54 & 0.53 & 1.9 & 2.1 & 1.84 & 0.6 & 3.4 & 2 & 1.2 & 0.148 & 0.014 & 0.02 & 0.131 & 0.04 \\
S36 & 7.18 & 2.39 & 18.5 & 12.1 & 0.9 & 0.7 & 4.1 & 20.1 & 9.2 & 0.155 & 0.013 & 0.02 & 0.036 & 0.038 \\
\hline
\end{tabular}

Note: All units are in $\mathrm{mg} / \mathrm{l}$ except $\mathrm{pH} \& \mathrm{EC}$

Table 7: Normal Statistic of Water Quality Parameters of Groundwater Samples (Post-Monsoon Season)

\begin{tabular}{|c|c|c|c|c|c|c|c|c|c|c|c|c|c|c|}
\hline Parameter & $\mathrm{pH}$ & $\mathrm{EC}$ & $\mathrm{Ca}$ & $\mathrm{Mg}$ & $\mathrm{Na}$ & $\mathrm{K}$ & $\mathrm{HCO}_{3}$ & $\mathrm{Cl}$ & $\mathrm{SO}_{4}$ & $\mathrm{Fe}$ & $\mathrm{Mn}$ & $\mathrm{Cu}$ & $\mathrm{Zn}$ & $\mathrm{Ni}$ \\
\hline Min & 7.11 & 0.42 & 1.9 & 1.2 & 0.08 & 0.09 & 0.5 & 2 & 1.1 & 0.127 & 0.002 & 0.003 & 0.002 & 0.02 \\
\hline MAX & 8.54 & 6.13 & 35 & 22.1 & 2.95 & 0.89 & 8.9 & 34.5 & 19.8 & 0.267 & 0.09 & 0.24 & 0.131 & 0.128 \\
\hline AVG & 7.713 & 2.161 & 13.231 & 6.758 & 0.931 & 0.301 & 3.683 & 12.063 & 6.175 & 0.204 & 0.017 & 0.029 & 0.056 & 0.057 \\
\hline MED & 7.82 & 1.875 & 11.45 & 5.1 & 0.855 & 0.2 & 3.4 & 8.8 & 4.7 & 0.232 & 0.013 & 0.015 & 0.053 & 0.050 \\
\hline SD & 0.403 & 1.315 & 7.940 & 4.916 & 0.695 & 0.236 & 2.029 & 8.108 & 4.011 & 0.051 & 0.016 & 0.042 & 0.031 & 0.025 \\
\hline
\end{tabular}

Table 8: Correlation Matrix of Water Quality Parameters (Post-Monsoon Season)

\begin{tabular}{|c|c|c|c|c|c|c|c|c|c|c|c|c|c|c|}
\hline Parameter & $\mathrm{Ph}$ & EC & $\mathrm{Ca}$ & $\mathrm{Mg}$ & $\mathrm{Na}$ & $\mathrm{K}$ & $\mathrm{HC} 03$ & $\mathrm{CI}$ & $\mathrm{SO}_{4}$ & $\mathrm{Fe}$ & $\mathrm{Mn}$ & $\mathrm{Cu}$ & $\mathrm{Zn}$ & $\mathrm{Ni}$ \\
\hline $\mathrm{pH}$ & 1 & & & & & & & & & & & & & \\
\hline EC & -0.50451 & 1 & & & & & & & & & & & & \\
\hline $\mathrm{Ca}$ & -0.53341 & 0.966759 & 1 & & & & & & & & & & & \\
\hline $\mathrm{Mg}$ & -0.50948 & 0.936711 & 0.869756 & 1 & & & & & & & & & & \\
\hline $\mathrm{Na}$ & 0.152483 & 0.417972 & 0.327701 & 0.375489 & 1 & & & & & & & & & \\
\hline $\mathrm{K}$ & -0.266 & 0.596688 & 0.576468 & 0.551492 & 0.439325 & 1 & & & & & & & & \\
\hline $\mathrm{HC} 03$ & -0.33482 & 0.785863 & 0.725173 & 0.727647 & 0.652639 & 0.531902 & 1 & & & & & & & \\
\hline $\mathrm{CI}$ & -0.50441 & 0.966315 & 0.973868 & 0.9148 .3 & 0.343169 & 0.596896 & 0.715447 & 1 & & & & & & \\
\hline $\mathrm{SO}_{4}$ & -0.5137 & 0.94783 & 0.906905 & 0.914573 & 0.364497 & 0.649377 & 0.683908 & 0.900884 & 1 & & & & & \\
\hline $\mathrm{Fe}$ & -0.30611 & 0.333804 & 0.372435 & 0.183854 & 0.131454 & 0.15554 & 0.205748 & 0.339897 & 0.307754 & 1 & & & & \\
\hline $\mathrm{Mn}$ & -0.11617 & 0.409401 & 0.384323 & 0.450269 & 0.279814 & -0.13227 & 0.363421 & 0.393856 & 0.311428 & 0.041928 & 1 & & & \\
\hline $\mathrm{Cu}$ & -0.05328 & 0.197615 & 0.223106 & 0.202715 & 0.131923 & 0.132868 & 0.095729 & 0.262135 & 0.089359 & 0.152835 & 0.20543 & 1 & & \\
\hline $\mathrm{Zn}$ & 0.17389 & -0.24795 & -0.27466 & -0.22673 & 0.070478 & 0.007933 & 0.082375 & -0.30875 & -0.23838 & -0.24947 & -0.19863 & 0.073794 & 1 & \\
\hline $\mathrm{Ni}$ & -0.34464 & 0.23942 & 0.287547 & 0.129768 & -0.06787 & 0.1282 & 0.31724 & 0.240316 & 0.119644 & 0.286241 & -0.02933 & 0.187908 & 0.055865 & 1 \\
\hline
\end{tabular}

In Post monsoon: Table 8 shows highly positive correlation is observed between $\mathrm{Cl}^{-}$and $\mathrm{Ca}^{++}(0.974), \mathrm{Ca}$ and $\mathrm{EC}(0.967), \mathrm{Cl}^{-}$ and $\mathrm{EC}(0.966), \mathrm{SO}_{4}$ and $\mathrm{EC}(0.948)$. Where, highly negative correlation is observed between $\mathrm{Ca}$ and $\mathrm{pH}(-0.533)$. Very poor positive correlation was observed between $\mathrm{Zn}$ and $\mathrm{K}$.

\section{Conclusion}

In the present study water quality has been calculated to assess the suitability of groundwater for drinking purpose along the periphery of, Kopargaon, Maharashtra, India. Better water quality was found in the post monsoon season than that of pre-monsoon season because of inflow of freshwater. The results shows that the concentration of alkalinity, total hardness, $\mathrm{Ca}, \mathrm{Mg}, \mathrm{Cl}$ at most of the sampling stations having higher values than the permissible limit prescribed by the Indian standards. In the present study, the correlation of physico-chemical parameters of groundwater revealed that all the parameters were more or less correlated with one another. Groundwater of the study area shows alkaline earth (ca and $\mathrm{mg}$ ) exceeds than alkalis ( $\mathrm{Na}$ and $\mathrm{K}$ ), weak acids ( $\mathrm{HCO} 3$ ) exceeds than strong acids $(\mathrm{Cl}, \mathrm{SO} 4$ and $\mathrm{NO} 3)$. The $\mathrm{Ca}, \mathrm{Mg}$ and $\mathrm{HCO} 3$ indicate hardness is dominated by the alkaline earth and weak acids. The water increases its major ions and electrical conductivity in pre monsoon period as compared to post monsoon period due to over exploitation of groundwater. From correlation analysis it was observed that very strong correlation exist between $\mathrm{HCO}_{3}$ and $\mathrm{Na}(0.961), \mathrm{HCO}_{3}$ and $\mathrm{Mg}^{++}(0.935), \mathrm{HCO}_{3}$ and $\mathrm{EC}$ (0.927). Where highly negative correlation is observed between $\mathrm{Na}$ and $\mathrm{pH}(-0.538)$ during pre-monsoon season. During post monsoon season highly positive correlation is observed between $\mathrm{Cl}^{-}$and $\mathrm{Ca}^{++}(0.974), \mathrm{Ca}$ and $\mathrm{EC}(0.967), \mathrm{Cl}^{-}$and $\mathrm{EC}(0.966), \mathrm{SO}_{4}$ and EC (0.948). Where, highly negative correlation is observed between $\mathrm{Ca}$ and $\mathrm{pH}(-0.533)$. The analysis shows that the groundwater of the study area need some treatment before it consumption. It is recommended that water analysis should be carried out from time to time to monitor the rate and kinds of contamination.

\section{Acknowledgements}

The author would like to acknowledge the cooperation from Department of Applied Geology, School f Environmental \& Earth Sciences, North Maharashtra University Jalgaon, India.

\section{References}

[1] APHA (1998) Standard methods for the examination of water and waste water, 19th edition APHA, Washington DC, USA.

[2] Dr.C.Gajendran, S.Jayapriya, Diana Yohannan, Oshin Victor, Christina Jacob, (2013), "Assessment of groundwater quality in Tirunelveli District, Tamil Nadu, India", International journal of environmental sciences, Volume 3, No 6, pp.1874-1880.

[3] Gajendran.C., and Thamarai.P., (2008), Study on statistical relationship between ground water quality parameters in Nambiyar River basin, Tamil Nadu, India, International journal on pollution research, 27(4), pp. $679-683$.

[4] Golekar R B, Baride M V and Patil S. N. (2013) Groundwater appraisal of shallow and deep aquifers from Anjani and Jhiri river Catchment, Northern Maharashtra India SRTMU's Research Journal of Science 2(2) pp. $118-129$.

[5] Ground Water Information Ahmednagar District Maharashtra, (2010), Government of India, Ministry of Water Resources, Central ground Water Board, pp. 1-27.

[6] Karanth, K. R., (1987). Groundwater assessment, development and management, Tata-McGraw Hill Publishing Company Limited, New Delhi.

[7] Khwaja M. Anwar and Aggarwal Vanita, (2014), Analysis of groundwater quality using statistical techniques: A case study of Aligarh city (India), International journal of technical research and applications-ISSN: 2320-8163, Volume 2, Issue 5, pp.100-106.

[8] Mondal, N. C., Saxena, V. K., and Singh, V. S. (2005) Assessment of groundwater pollution due to tannery industries in and around Dindigul, Tamilnadu, India Environmental Geology 48 (2): 149_ 157 http://dx.doi.org/10.1007/s00254-005-1244-z.

[9] Patil, S. N., Rokade, V. M., Patil, Sandeep P., Patil Sachin., and Jagadale Shantaram (2010) Hydrogeochemical Investigation of Groundwater from Faizpur area of Jalgaon District, Maharashtra, Journal of Applied Geochemistry 12 (2): 217-223.

[10] Shashi Kant, Singh Y.V., Kumar Lokesh Jat, R.Meena and Singh S.N. (2015), Assessment of ground water quality of lahar block, Bhind District in Madhya Pradesh, Vol.3, and International Journal of Advanced Geosciences pp.38-41. http://dx.doi.org/10.14419/ijag.v3i2.4949. 
[11] Yadav,R.K.(2012) Correlation Of Physico-Chemical Analysis Of Ground Water Of Jaipur City (Rajasthan, India) International journal of Science and Nature, 3(4): 923-924.

[12] S. M. Mazhar Nazeeb Khan* and A. Ravi Kumar (2012)" Interpretation of Groundwater Quality using Correlation and Linear" Regression Analysis from Tiruchengode Taluka, Namakkal district, Tamilnadu, India "Journal of Chemical and Pharmaceutical Research ,ISSN : 0975-7384 pp. 4514-4522.

[13] R. S. Sapkal1, Dr. S. S. Valunjkar (2013)'Development and Sensitivity Analysis Of Water QualityIndex For Evaluation Of Surface Water For Drinking Purpose" International Journal of Civil Engineering And Technology (IJCIET) ISSN 0976 - 6308 spp. 119-134.

[14] Suman Pawar, Srivastava. R.K" (2012) Assessment of groundwater quality in contiguous of integrated industrial estate Pantagar, Uttarakhand" International Journal of Environmental Sciences ISSN 0976 - 4402pp1130-1140.

[15] Babak Jafari Salim1, Gholamreza Nabi Bidhendi1, Amir Salemi2, Masoud Taheryioun1, Mojtaba Ardestani1, (2009), "Water Quality Assessment of Gheshlagh River UsingWater Quality Indices", Environmental Sciences Vol.6, No.4, Summer pp. 19-28.

[16] M. Sherif a, M. Mohamed a,b, A. Kacimov c, A.Shetty (2011)" Assessment of groundwater quality in the northeastern coastal area of UAE as precursor for desalination "Science Direct pp. 436-446.

[17] S.Venkateswarana and S.Deepaa "Assessment of Groundwater Quality using GIS Techniques in Vaniyar Watershed, Ponnaiyar River, Tamil Nadu" Science Direct, pp. 1283 - 1290.

[18] Megersa Olumana Dinkaa,, Willibald Loiskandlb, Julius Musyoka Ndambukic" Hydrochemical characterization of various surface water and groundwater resources available in Matahara areas, Fantalle Woreda of Oromiya region" Journal of Hydrology: Regional Studies , pp. 444-456.

[19] Amina Ramdani a, Haja Mebarka Djellouli a, Nadia Aï Yala a, Safia Taleb a, Abderazzak Benghalem a, Cherif Mahib, Abderazzak Khadraoui, (2012), "Physico-Chemical Water Quality in Some Regions of Southern Algeria and Pretreatment Prediction" Science Direct pp. $335-339$.

[20] KG Rosina, Ravinder Kaura, SD Singhb, P Singhb, DS Dubeyb "Groundwater vulnerability to contaminated irrigation waters A case of peri-urban agricultural lands around an industrial district of Haryana, India" Science Direct, pp 200-210. 